\title{
Clareamento dentário durante tratamento ortodôntico
}

\author{
Dental bleaching during orthodontic treatment
}

\author{
Ana Lúcia Alves de Oliveira Freire* \\ Marlus da Silva Pedrosa** \\ Marcus Barreto Vasconselos ${ }^{* * *}$ \\ Maura Régia Lima Verde Moura Lopes ${ }^{* * *}$ \\ Rianny Maria Barros Lopes ${ }^{* * * *}$ \\ Maria Reggiani Azevedo Carvalho ${ }^{* * * * *}$
}

\section{Resumo}

Objetivo: revisar a literatura atualmente disponível sobre clareamento dentário durante o tratamento ortodôntico. Revisão de literatura: uma pesquisa bibliográfica exploratória foi realizada na base de dados eletrônica Public Medline (PubMed) empregando os termos de busca "dental bleaching" ou "tooth whitening" e "orthodontics". Os critérios de inclusão foram: artigos de pesquisas originais e ensaios clínicos, publicados em português, espanhol ou inglês. Não foram aplicados limites à data de publicação. A busca resultou em um total de três estudos, publicados em 2011, 2014 e 2016. Há evidência de estudos clínicos que ressaltam a eficácia do clareamento dentário em pacientes que usam aparelho ortodôntico. Outro estudo mostra diferenças significativas entre a cor geral do dente e a da superfície abaixo dos braquetes ortodônticos. Considerações finais: há necessidade de estudos com metodologias acuradas, a fim de que se possam fornecer dados mais precisos para alicerçar recomendações clínicas, técnicas e materiais, quando se considera essa abordagem de tratamento.

Palavras-chave: Clareamento dental. Estética dentária. Ortodontia.

\section{Introdução}

O sucesso do tratamento ortodôntico com aparelhos fixos depende de vários fatores, dentre os quais, destacam-se a colagem adequada de braquetes ortodônticos e a longevidade desses acessórios nos dentes ${ }^{1}$, sendo essencial manter a integridade e a estabilidade biomecânicas da interface braquete/adesivo, que transfere a força gerada pelos arcos para os dentes ${ }^{2}$.

A perda dos braquetes durante o tratamento ortodôntico é preocupante para os ortodontistas e requer tempo e despesa adicionais no consultório, para substituir braquetes perdidos, bem como para estender o tempo de tratamento em alguns casos ${ }^{3,4}$. Tratamentos odontológicos mediante a utilização de agentes clareadores podem afetar a adesão dos braquetes à superfície devido à presença de solventes e outros componentes que podem aumentar a solubilidade ou a degradação do suporte adesivo ${ }^{1}$.

Os efeitos do clareamento dentário na superfície do esmalte e na resina composta foram amplamente avaliados e, apesar de conflitantes, mostram que o clareamento dentário antes da colagem dos braquetes pode interferir na adesão dos braquetes aos dente, dependendo do intervalo de tempo entre a realização desses procedimentos ${ }^{1,5}$.

Graduada em Odontologia no Centro Universitário Uninovafapi e pós-graduanda em Ortodontia no Instituto Lato Sensu, Teresina, PI, Brasil.

Graduado Odontologia, Faculdade Integral Diferencial, DeVry, Facid, Teresina, PI, Brasil.

Especialista em Ortodontia e Ortopedia Facial, ABO. Mestre em Odontologia (Ortodontia), Universidade Ceuma. Professor e coordenador do Programa de Pós-Graduação em Ortodontia do Instituto Lato Sensu. Diretor científico na mesma instituição, Teresina, PI, Brasil.

Especialista em Ortodontia e Ortopedia Facial, ABO. Mestre em Odontologia, São Leopoldo Mandic. Doutora em Ciências Odontológicas (Ortodontia), São Leopoldo Mandic. Professora e coordenadora do Programa de Pós-Graduação em Ortodontia do Instituto Lato Sensu, Teresina, PI, Brasil.

${ }_{* * * * *}$ Especialista em Ortodontia e Ortopedia Facial, Centro Universitário Uninovafapi. Mestre em Odontologia pela Universidade Federal do Piauí. Professora do Programa de Pós-Graduação em Ortodontia do Instituto Lato Sensu, Teresina, PI, Brasil.

******* Especialista em Ortodontia e Ortopedia Funcional dos Maxilares, ABO. Mestre em Odontologia (Ortodontia) pela Universidade Ceuma. Docente do Programa de Pós-Graduação em Ortodontia do Instituto Lato Sensu, Teresina, PI, Brasil. 
De acordo com Consolar et al. ${ }^{6}(2013)$, desprendimento dos braquetes, manchas brancas, perda de uniformidade na cor do dente após remoção do aparelho ortodôntico e retenção de placa bacteriana estão entre as consequências do clareamento dentário associado ao tratamento ortodôntico, uma vez que os agentes clareadores promovem a desmineralização do esmalte. Entretanto, em face da crescente popularização da prática de clareamento dentário durante o tratamento ortodôntico, há poucas evidências científicas sobre essa temática.

Existe um risco alto de que o clareamento dentário durante o tratamento ortodôntico possa ocasionar manchas e irregularidades na cor da superfície dentária após a remoção dos braquetes, e a relação custo-benefício pode não ser favorável. Assim, associar o clareamento dentário com tratamento ortodôntico requer muita cautela e conhecimento científico. Além disso, é de suma importância que profissionais e pacientes estejam cientes de seus potenciais riscos ${ }^{6}$. Nesse sentido, o presente trabalho objetivou revisar a literatura atualmente disponível sobre clareamento dentário realizado durante o tratamento ortodôntico.

\section{Revisão de literatura}

\section{Estratégia de busca}

Para o propósito deste estudo, uma busca bibliográfica exploratória foi realizada na base de dados eletrônica Public Medline (PubMed) empregando os termos de busca combinados: "dental bleaching" ou "tooth whitening" e "orthodontics".

Os critérios de inclusão foram: artigos de pesquisas originais e ensaios clínicos, publicados em português, espanhol ou inglês, sem limites quanto à data de publicação. Títulos e resumos foram selecionados por relevância, considerando os critérios de inclusão. Em seguida, foi obtido o texto completo dos estudos listados e selecionados com os mesmos critérios, sendo os elegíveis incluídos nesta revisão.

Foram desconsiderados manuscritos em idiomas não selecionados para este estudo, artigos não disponíveis em texto completo e publicações não relevantes/condizentes com a temática proposta. Os estudos elegíveis também tiveram as suas listas de referência selecionadas.

\section{Resultados}

Segundo os critérios de inclusão e exclusão mencionados na metodologia, para esta revisão, a busca resultou em um total de três publicações ${ }^{1,7,8}$ mediante a busca eletrônica na base de dados PubMed. Segundo nossos resultados, os estudos foram publicados em $2011^{7}, 2014^{8}$ e $2016^{1}$. A seguir, são apresentados os resultados encontrados sobre clare- amento dentário em pacientes utilizando aparelho ortodôntico.

Jadad et al. ${ }^{7}$ (2011) avaliaram a eficácia de um agente clareador novo (peróxido de hidrogênio a 8\%) em pacientes em uso de aparelho ortodôntico fixo. Para tal, foram avaliados seis dentes superiores de 40 pacientes com idades entre 18 e 40 anos. Os pacientes foram divididos em dois grupos (20 em cada grupo): no grupo A, os pacientes utilizaram agente clareador durante o tratamento ortodôntico; no grupo B, os pacientes utilizaram agente clareador após o tratamento ortodôntico. Para cada grupo, registrou-se a cor inicial do dente com uma matriz de polivinilsiloxano e um espectrofotômetro. Todos os grupos foram submetidos ao tratamento clareador com peróxido de hidrogênio a 8\% (Opalescence Treswhite Ortho, Ultradent, Opal Orthodontics, South Jordan, Utah) durante 10 dias, em sessões de 45 minutos. Para o grupo A, o tratamento foi realizado 10 dias antes de terminar o tratamento ortodôntico. Participantes do grupo B tiveram o mesmo protocolo de clareamento após a remoção dos braquetes. Para o grupo sem braquetes, a alteração da cor variou de 3 a 12 (média, 8; mediana, 8,3). Para o grupo com braquetes, o intervalo foi entre 4 e 13 (média, 9; mediana, 8,5). Os resultados indicam clareamento significativo de dentes em ambos os grupos, com e sem braquetes ( $>0,05$ ). Assim, o agente clareador empregado mostrou-se eficiente em pacientes em uso de aparelhos ortodônticos fixos.

Em estudo com o objetivo de avaliar a eficácia do clareamento dentário sob o braquete ortodôntico, Lunardi et al. ${ }^{8}$ (2014) realizaram estudo com blocos $(8 \mathrm{~mm} \times 8 \mathrm{~mm})$ contendo coroa de incisivos bovinos $(\mathrm{n}=32)$. A coloração de blocos de dentes com chá preto foi realizada durante seis dias. Os 32 blocos foram distribuídos aleatoriamente em 4 grupos: 1) clareamento caseiro com braquete; 2) clareamento caseiro sem braquete; 3 ) clareamento de consultório com braquete; e 4) clareamento de consultório sem braquete. A avaliação de cor foi realizada usando espectrofotômetro de refletância de cor. Os grupos 1 e 2 foram submetidos ao clareamento com peróxido de carbamida a 15\%, 4 horas por dia, durante 21 dias. Os grupos 3 e 4 foram submetidos a 3 sessões de tratamento de clareamento em consultório com peróxido de hidrogênio a $38 \%$. Após a remoção dos braquetes, uma segunda avaliação de cor foi realizada, a fim de verificar a diferença entre a área sob o braquete e no torno dele e, depois de 7 dias, para verificar a estabilidade da cor. A análise dos dados foi realizada utilizando o teste t pareado e a análise de variância bidirecional e de Tukey. Os resultados mostraram que a técnica de clareamento caseiro é mais eficaz em comparação ao clareamento de consultório. Houve uma diferença significativa entre a margem e os valores de cor central das amostras coladas com braquetes. Assim, a colagem dos braquetes afetou a efetividade de ambas as modalidades de clareamento. 
Montenegro-Arana et al. ${ }^{1}$ (2016), em estudo randomizado duplo-cego, avaliaram a sensibilidade dentária e a eficácia de dois tipos de agentes clareadores (Treswhite Ortho [TWO] e Treswhite Supreme [TWS]) em pacientes em uso de aparelhos ortodônticos. Para tal, 40 pacientes entre 18 e 40 anos de idade foram aleatoriamente divididos em dois grupos $(n=20)$, de acordo com o agente clareador aplicado. A cor dos seis dentes anteriores superiores foi avaliada com um espectrofotômetro antes e após o clareamento dentário. A sensibilidade dentária foi avaliada em três escalas antes e durante o clareamento. As escalas para sensibilidade utilizadas foram: uma escala de cinco pontos que varia de 0 (ausência de sensibilidade) a 4 (sensibilidade severa), uma escala numérica de 0 a 100 (NRS 101) e uma escala visual analógica. No que diz respeito à eficácia do clareamento, foi encontrada uma redução significativa (variando de 7.3-9.6 a 5.3-9.5 unidades de escala Vita para TWO e TWS, respectivamente, $\mathrm{p}<0,001$ ), não havendo diferença estatística entre os grupos ( $p>0,63)$. O número de pacientes com sensibilidade foi de $58,8 \%$ e de $73,3 \%$ nos grupos TWO e TWS, respectivamente $(\mathrm{p}=0,53)$; no entanto, com cada uma das três escalas utilizadas, a intensidade de sensibilidade foi baixa, e não houve diferença estatística entre TWO e TWS ( $>>0,05)$. Os autores concluíram que, apesar de produzir efeito colateral de baixa sensibilidade dentária, os dois tipos de agentes clareadores foram eficazes em pacientes com aparelhos ortodônticos fixos.

\section{Discussão}

A satisfação do paciente com a aparência dentária é afetada pela cor e pelo posicionamento dos dentes, havendo o desejo ou a necessidade de restaurações estéticas e de tratamento ortodôntico. Reconhecer os fatores que afetam a satisfação dos pacientes pode orientar a atitude do profissional em relação aos tratamentos propostos, de forma a melhorar a estética dentária9 ${ }^{9}$.

Como a maioria dos pacientes que usa aparelhos ortodônticos percebe alterações na cor dos dentes, é importante que sejam fornecidas juntamente com 0 tratamento ortodôntico a satisfação estética e a possibilidade de os dentes terem uma cor socialmente aceitável durante o tratamento ${ }^{7}$. É provável que a maioria dos ortodontistas encontre pacientes que solicitem produtos e procedimentos de clareamento, sendo prudente a familiaridade do profissional com a literatura acerca do clareamento ${ }^{10,11}$.

Segundo os resultados dessa revisão de literatura, existem evidências divergentes. Há evidência de estudos clínicos ${ }^{7}$ e de estudos randomizados $\operatorname{cegos}^{1}$ que ressaltam a eficácia do clareamento dentário em pacientes em uso de aparelho ortodôntico. Entretanto, outro estudo ${ }^{8}$ mostra diferenças significa- tivas entre a cor geral do dente e a superfície abaixo dos braquetes ortodônticos. Essa diferença pode ser explicada devido às diversas metodologias adotadas: os dois primeiros estudos visaram ao clareamento dentário de forma geral, enquanto o último verificou também o clareamento dentário abaixo do braquete.

Mesmo havendo preocupação entre os profissionais quanto à sua efetividade ${ }^{12}$, o clareamento dos dentes é possível durante o tratamento ortodôntico com aparelhos fixos sem perda estética ${ }^{1,7,12}$, uma vez que o agente clareador pode se difundir por meio das microestruturas dos tecidos dentários ${ }^{12}$. Nesse caso, o clareamento dentário também pode atuar como fator motivador, evitando a desistência do paciente ou a interrupção do tratamento ${ }^{12}$. Além disso, o peróxido de hidrogênio poderia exercer um efeito positivo no controle de placa e na redução de gengivite ou doenças periodontais durante 0 tratamento ${ }^{13}$.

Entretanto, como demonstrado nesta revisão de literatura, não existem evidências suficientes acerca da possibilidade de clareamento dentário durante o tratamento ortodôntico ${ }^{14}$. Assim, apesar de a maioria dos resultados levantados corroborar com resultados clínicos satisfatórios ${ }^{7,1}$, estes não devem ser extrapolados, uma vez que é provável que existam contínuas divergências sobre recomendações clínicas, técnicas e de materiais, quando se considera essa abordagem de tratamento.

Tal fato se dá pela carência de estudos clínicos para pautar, por meio de evidência científica, a decisão de se proceder ao clareamento dentário durante o tratamento ortodôntico. Além disso, essa abordagem requer cautela e avaliação individual de cada paciente, devendo a tomada de decisão de proceder ou não com o clareamento ser pautada nas evidências científicas disponíveis, combinadas com a experiência clínica do profissional.

\section{Considerações finais}

A literatura é conflitante, havendo evidências clínicas satisfatórias acerca da eficácia do clareamento dentário em pacientes em uso de aparelho ortodôntico, bem como resultados que não suportam tal conduta.

Não obstante, esses resultados não devem ser extrapolados, havendo a necessidade de estudos com metodologias acuradas, a fim de que se possa fornecer dados mais precisos para alicerçar recomendações clínicas, técnicas e materiais, quando se considera essa abordagem de tratamento.

$\mathrm{O}$ número de estudos publicados sugere a necessidade de estudos clínicos futuros in vitro e in vivo com metodologias acuradas, para pautar, por meio de evidências científicas, essa abordagem de tratamento, considerando a preocupação estética na atualidade. 


\section{Abstract}

Objective: to review the literature currently available on the scientific evidence on dental bleaching during orthodontic treatment. Literature review: an exploratory bibliographic search was performed through Public Medline (PubMed) using the search terms "dental bleaching" or "tooth whitening" and "orthodontics". Inclusion criteria were original research articles and clinical trials published in Portuguese, Spanish and English. No limits were applied to the date of publication. The search resulted in three studies published in 2011, 2014 and 2016. There is evidence of clinical studies that highlight the effectiveness of tooth bleaching in patients wearing orthodontic appliances. However, another study shows significant differences between the color of the tooth and the surface below the orthodontic brackets. Final considerations: there is a need for studies with accurate methodologies, in order to provide data to support clinical, technical and material recommendations when considering this treatment approach.

Keywords: Dental esthetics. Orthodontics. Tooth bleaching.

\section{Referências}

1. Montenegro-Arana A, Arana-Gordillo LA, Farana D, Davila-Sanchez A, Jadad E, Coelho U et al. Randomized Double-blind Clinical Trial of Bleaching Products in Patients Wearing Orthodontic Devices. Oper Dent 2016; 41(4):379-87.

2. Eliades T, Brantley WA. The inappropriateness of conventional orthodontic bond strength assessment protocols. Eur J Orthod 2000; 22(1):13-23.

3. Oesterle LJ, Shellhart WC. Effect of aging on the shear bond strength of orthodontic brackets. Am J Orthod Dentofacial Orthop 2008; 133(5):716-20.

4. Ray S, Londhe S, Mitra R, Chopra SS. bleaching and desensitizing agents contraindication for patients seeking orthodontic treatment? Orthodontics (Chic.) 2012; 13(1):e181-7.

5. Rego MV, dos Santos RM, Leal LM, Braga CG. Evaluation of the influence of dental bleaching with $35 \%$ hydrogen peroxide in orthodontic bracket shear bond strength. Dental Press J Orthod 2013; 18(2):95-100.

6. Consolaro A, Consolaro RB, Francischone L. Clarifications, guidelines and questions about the dental bleaching "associated" with orthodontic treatment. Dental Press J Orthod 2013; 18(5):4-10.

7. Jadad E, Montoya J, Arana G, Gordillo LA, Palo RM, Loguercio AD. Spectrophotometric evaluation of color alterations with a new dental bleaching product in patients wearing orthodontic appliances. Am J Orthod Dentofacial Orthop 2011; 140(1):e43-7.

8. Lunardi N, Correr AB, Rastelli AN, Lima DA, Consani RL. Spectrophotometric evaluation of dental bleaching under orthodontic bracket in enamel and dentin. J Clin Exp Dent 2014; 6(4):e321-6.

9. Maghaireh GA, Alzraikat H, Taha NA. Satisfaction with Dental Appearance and Attitude toward improving Dental Esthetics among Patients attending a Dental Teaching Center. J Contemp Dent Pract 2016; 17(1):16-21.

10. Thickett E, Cobourne MT. New developments in tooth whitening. The current status of external bleaching in orthodontics. J Orthod 2009; 36(3):194-20.
11. Slack ME, Swift EJ Jr, Rossouw PE, Phillips C. Tooth whitening in the orthodontic practice: a survey of orthodontists. Am J Orthod Dentofacial Orthop 2013; 143(4 Suppl):S64-71.

12. Gomes MN, Dutra H, Morais A, Sgura R, Devito-Moraes AG. In-Office Bleaching During Orthodontic Treatment. J Esthet Restor Dent 2017; 29(2):83-92.

13. Lazarchik DA, Haywood VB. Use of tray-applied 10 percent carbamide peroxide gels for improving oral health in patients with special-care needs. J Am Dent Assoc 2010; 141:639-46.

14. Márquez JF, Pedroza-Garcés A, Villada-Castro M. Aclaramiento dental, durante y después de ortodoncia. Rev CES Odont 2012; 25(2):54-62.

Endereço para correspondência:

Marlus da Silva Pedrosa

Endereço: Rua Senador Joaquim Pires, 723

CEP 64049-590, Teresina, Piauí, Brasil

Telefone: (86) 99492-4142

E-mail: marluspedrosa@gmail.com

Recebido: 21/11/17. Aceito: 19/12/17. 